Kurmo Konsa

\title{
MODERN CONSERVATION: CONNECTING OBJECTS, VALUES AND PEOPLE
}

The past exists today in the form of objects, memories and landscapes. Of course this existence is illusory; objects, memories or landscapes have not remained the same throughout time. The past can still only exist in people's heads and nowhere else. At the same time, people constantly require objects that recall the presence of the past. It is difficult to foresee the future, but it can be created. One of the most important materials available to us for building the future is the past. The future not only depends on the past, but it is literally built on the past. Heritage is a technique that must be used as effectively as possible to resolve the local and global problems of societies today and in the future. If we do not do so, heritage will become unnecessary, at least in the form that it had developed by the late $18^{\text {th }}$ century and is still in use today. ${ }^{1}$ Heritage is a technique that we use today to create the present and the past and it depends on our current choices rather than on the past. Heritage is only a tool, but we should not forget that it is a very powerful tool for making these choices and implementing the decisions based thereon.

Heritage is always the carrier of definite values and meanings. Educing these values and taking them into account forms the basis for the management of the entire heritage process. According to the contemporary approach to heritage, the physical and spiritual aspects of heritage are

DOI: http://dx.doi.org/10.12697/BJAH.2015.10.03

Translated by Juta Ristsoo.

1 See for example, Kurmo Konsa, "Heritage as a Socio-Cultural Construct: Problems of Definition", Baltic Journal of Art History, 6 (2013), 123-149. 
inseparable. However, in practical heritage management, the main attention is still focused on the material side. There is also a clear reason for this, since the material side of heritage is easier to perceive and manage. The successful preservation of heritage and its utilisation in society depends to great degree on whether a conceptual framework is created for it that combines the spiritual and material side of heritage. Designing the bases for such a conceptual framework is the goal of this article. To achieve this, I will connect the main approaches to conservation (object-, value- and people-based methods) with the corresponding information models. I propose that information content models are central to object-based conservation and naturally value-based conservation relies on typologies. And I associate people-based conservation with ideas related to information ecology. The entire conservation process is based on knowledge and concepts and documentation is the tool that helps to record, collect and mediate this information. Therefore, the conservation process can be treated as an information process. The article provides a clearer conceptual framework for the co-treatment of the spiritual and material aspects of heritage.

\section{THE CONSERVATION OF HERITAGE OBJECTS}

Conservation is one way to preserve the objects that are part of heritage. Conservation as a profession as we know it today developed during the $19^{\text {th }}$ century. People have dealt with reconditioning, repairing and rebuilding the objects related to heritage since prehistoric times. However, all this is very far from conservation as we understand it today. In the $19^{\text {th }}$ century, conservation started to be seen as an integrated process comprised of several parts, including the scientific examination of objects; the determination and elimination of damaging factors (if this is possible of course); the creation of suitable storage conditions; the prevention of further deterioration; and the constant monitoring of the objects' condition. ${ }^{2}$ The processing of the objects has always been central to conservation. Of course, what is done specifically depends on the objects, as well as the goals of the processing. It is clear that conserving a building differs from restoring a painting and the conservation of an

2 For information on the history of conservation, see Jukka Jokilehto, A History of Architectural Conservation (Oxford, Auckland et al.: Butterworth-Heinemann, 2002). 
Old Believer's prayer book that is used everyday differs from the processing of parchment records that are stored in archives. Objects have both physical substance (they are things) as well as cultural meaning (they refer to, mean something). Understanding the connections between the material and immaterial aspects of objects forms the basis for the conservation process. This characteristic mixture of physical and cultural information is both the charm and burden of conservation. On the one hand, conservators use scientific research methods that provide scientific facts about the objects and on the other hand they try to understand the thoughts and attitudes of the people related to the objects. The dual nature of an object is related to the physical processing of the object and the interpretation of the object. The conservator deals with the physical side of the object, processes it, while, at the same time, interpreting it.

Along with the processing methods, various theoretical approaches, which provide the reasons for and explain the circulation of the heritage objects, are very important in conservation. The conservation approaches can be defined as being object-, value- or people-based, according to their focus. ${ }^{3}$ These approaches are not used in a definite temporal sequence, whereby they would preclude each other. Depending on the context, a specific temporal sequence does exist, but they are all in use today. The choice of the preferred approach depends on the goals of the method. These approaches express an increasingly inclusive and complex approach to conservation.

\section{OBJECT-BASED CONSERVATION}

In the object-based approach, it is naturally the object itself that is the focus of the conservation process. What is done with the object depends primarily on its condition and possible damage. At first glance, it may seem that the condition of an object or building is a state that is subordinated to so-called "objective" scientific analysis. However the reality is something different. The determination of condition presumes that an entire series of decisions, all of which are affected by the context and the connections to the object's function, objective and utilisation. Whenever object-based conservation theory is employed, it is important

3 Dean Sully, "Conservation Theory and Practice: Materials, Values and People in Heritage Conservation”, The International Handbooks of Museum Studies, 2, 13 (2013), 293-314. 
to preserve the material side of the heritage object. Therefore the physical damage suffered by an object also means that information about the past has been lost. The objective of the processing is the integrated physical preservation of the object, which is based on scientific research. The language that is spoken is mostly the language of science and especially the natural sciences. The processing is managed by the ethical principles of conservation, including minimal intervention, reversibility, recyclability, etc. An attempt is made to define some objective physical truth in the object; however, this may again result in the traces of history being removed from the object. There are always many different ways to conserve an object. What are the reasons for choosing one method among many? Which presentation of the object should be preferred? The object itself will not provide us with the answers to these questions.

Today, the treatment of objects as phenomena related to various types of information is at the centre of the conservation theories that underlie object-based conservation. Despite the development of an information society and the increasingly extensive utilisation of digital data processing in both art and conservation, physical objects have not disappeared. Quite the opposite - the concept of objects has expanded and includes ever more information. Below, I will talk about what artefacts are like in an informational sense, i.e. what information they contain and are connected to. This is a fundamental question in both historical science as well as preservation, since this is the basis for all interpretation and preservation strategies. It is clear that various objects are the carriers of very different kinds of information. Various approaches to the description of information related to artefacts have been proposed by different authors. Below, I will utilise a model that differentiates artefacts based on three informational levels: structural information or structural attributes; functional information or functional attributes; and context. ${ }^{4}$

\section{Structural information}

Structural information includes all the physical (structural) attributes of the object, such as material, construction, design, sound, smell and taste. This is all information that can be perceived by the senses and exam-

4 Ian Hodder, The Archaeology of Contextual Meanings (Cambridge: CUP, 1987); Susan M. Pearce, Interpreting Objects and Collections (London: Routledge, 1994); Peter van Mensch, "Methodological museology; or, towards a theory of museum practice", Objects of Knowledge (London, Atlantic Highlands: The Atlone Press, 1990), 141-157. 
ined using physicochemical research methods. Usually the concept of an "object" or "artefact" is used in this meaning, i.e. in order to indicate the object in the physical sense. Objects the comprise heritage are comprised of very different materials and each material in turn has quite a complicated composition. Materials also have different physicochemical attributes. This means that the object as a combination of materials forms a complicated system. Naturally, one must also consider the fact that the composition of the materials has changed significantly in time. In turn, the damage suffered by the artefacts depends to a great degree on the physicochemical properties of the materials. The durability of the objects depends greatly on the materials from which they are made. Inorganic materials, such as stone, ceramics or bronze, are relatively durable and last a long time. Organic materials, such as wood, textiles, leather, etc. are less resilient and tend to decompose faster. Therefore knowing about the materials is extremely important from the standpoint of preservation. This is actually the first step for successful preservation - knowing the object; knowing of what and how the object has been made. Since materials are so important when dealing with artefacts, their preservation is very closely related to materials science. What material an object is made of and how it was made depends on the physical attributes of the materials, financial limitations and cultural influences. Since objects perform various functions, this places different demands on the materials used to produce them. The materials must be available and their utilisation for the designated purpose must pay off. The ideas related to the value and attributes of the material, which are characteristic of the culture, also affect their utilisation for making objects. ${ }^{5}$ The construction expresses the way in which an object is produced and the parts it is comprised of. The construction of objects can differ a great deal based on the materials that have become available, the changed technologies, but also changing fashions and consumer demands. The general shape of the object is primarily defined by its function and the materials used. ${ }^{6}$ The design characterises the appearance of the object - its form, colour, surface finishing, decoration, style, iconography, etc. Objects made of the same material that are of similar construction can have different designs. The exterior shape of the object is called its form. Objects that

5 Robert McGhee, "Ivory for the Sea Woman: The symbolic attributes of prehistoric technology", Interpreting Objects and Collections, ed. by Susan Pearce (London: Routledge, 1994) 59-66.

6 Chris Caple, Objects: Reluctant Witnesses to the Past (London, New York: Routledge, 2006), 7. 
have similar form attributes belong to the same style. Styles vary based on time and geography. Compared to the materials, construction and general shape, design is much more culturally defined. The decoration of objects requires expending resources and labour. There can be many reasons why this is still done: ${ }^{7}$ to increase the value of the objects; to increase the status and social position of the objects' owner; to express affiliation; or to enable the object to perform a certain function, such as a religious one. Letters, numbers, texts, etc. can also be found on objects that are not explicitly communicative. Examples include gravestones, coins, containers, items of clothing, etc. Markings that are placed on objects can refer to their names, producers, factories, places of manufacture and indicate the quality or price of the item. Labels are often affixed to parts of complicated objects that are comprised of many pieces.

A special group of objects, which often bears information, is packaging. Packaging can be treated as an independent artefact, which also comprises the material context of a given object. In the case of paintings, the frames bear extremely important information. ${ }^{8}$ Quite often, ownership markings are put on the items by their owners. The various important structural features of artefacts include sound, smell and taste. Examples of such objects include church bells, musical instruments, perfumes or foodstuffs. Indirect information is the information that the object has acquired unintentionally or is collected in the object during its lifespan. For example, indirect information includes the chemical composition of the materials; the content of microelements; the relationship between various oxygen or carbon isotopes; silicon algae in clay; air bubbles, fingerprints on ceramics, etc. This is information that is extremely important from the viewpoint of scientific research (the determination of the origin and authenticity, dating, technology analysis, etc.). In the course of the objects being used, all kinds of information collect in them. Objects wear as they are used; traces of the various substances they have come in contact with are left on them. The examination of the structural information related to artefacts is important in archaeology, ethnology, art history and the history of technologies. Indirect information helps to define the origin of the materials used to make the objects. Every

7 Ian Hodder, The Present Past (London: Batsford, 1982), 185.

8 See for example, Henry Heydenryk, The Art and History of Frames: An Inquiry Into the Enhancement of Paintings (New York: J. H. Heineman, 1963); Tim Newberry, Frames and Framing (London: Ashmolean Museum, 2006). 
contact between the object and the world affects the object and leaves its trace. These types of influences can be divided into three groups: (a) an external substance connected to the object - all kinds of substances that have ended up on the surface of the object; materials inside or absorbed into the objects. This can include the layers of soot on clay pots, food scraps left in dishes; plant samples in books; or (b) mechanical damage - scratches and dents, which are caused by contacts with other objects and materials; the surface wear of the object; traces of use, etc. And (c) chemical and biological damage - caused by external chemical substances or the impact of organisms.

\section{Functional information}

Functional information refers to the utilisation of the artefact. Understandably, each artefact can perform different functions. Function depends to a great extent on whether the artefact is utilitarian or communicative. Practical or utilitarian functions are connected to the physical use of an object. Objects are used in everyday life for a definite purpose, for instance a pencil is used for writing and a shovel for digging. Where does the information about the function of objects come from? To a certain extent, it is possible to deduce the function of objects from their shape and the materials used to produce them, but the majority of this information is acquired from written and pictorial sources. In addition to its practical function, the appearance of very many objects is also very important. This supplementary function, in addition to the practical one, is known as the aesthetic function. Every object that has been chosen or produced by a person has a definite shape, colour and texture. Thus, all objects have a certain aesthetic function. ${ }^{9}$ For works of art or phenomena that can be treated as art, the main function is usually the aesthetic one. All artefacts can have symbolic functions; they refer to some event, abstract idea or meaning, etc. that is located outside of the object. Objects mean something; they function as symbols and/or

9 Alois Riegl, “The Modern Cult of Monuments: Its Essence and its Development", Historical and Philosophical Issues in the Conservation of Cultural Heritage, ed. by Nicholas S. Price, Mansfield Kirby Talley Jr., Alessandra M. Vaccaro (Los Angeles: The Getty Conservation Institute, 1996), 69-83; Mansfield Kirby Talley Jr., "The Eye's Caress: Looking, Appreciation and Connoisseurship", Historical and Philosophical Issues in the Conservation of Cultural Heritage, 2-41. 
texts. ${ }^{10}$ Even the materials used to produce objects have a certain symbolic meaning. The meaning of objects and their symbolic function depend to a great degree on the culture and the given context. Owning certain objects indicates erudition, wealth, high social standing and prestige. Consequently, these objects also perform a value-related function. ${ }^{11}$ These types of objects include works of art, haute couture, weapons, etc. Certain materials, such as gold, silver, amber, furs and ivory, have been valued in almost all cultures and all time periods and the ownership of objects made of these materials indicates wealth and a dominant position in society. These materials are characterised by a beautiful appearance, durability and limited availability, which complicates their acquisition. ${ }^{12}$ The metaphysical meaning refers to the relations with the supernatural world that is attributed to many artefacts, such as grave goods, statues of gods, icons, and sacrificial items, various ritual items, etc. These objects are located between the visible and invisible world. They have properties lacked by ordinary artefacts and this makes them very valuable for believers. ${ }^{13}$ The possession and use of such sacred objects is very restricted and regimented. Thus, in some cultures, objects in which spirits live must be fed regularly. In most cases, the determination of metaphysical functions cannot rely only on the exterior attributes of the objects, exact information on the given culture is also necessary. The documentary value of objects and their treatment as sources of information did not become important until the $19^{\text {th }}$ century. Then, it became apparent that not only communicative artefacts but also all other objects bear information. William Morris particularly emphasised the importance of treating objects as witnesses to the past. ${ }^{14}$ The presentation of objects as sources of information is the primary task and function of memory institutions. Objects may cause emotional reactions in people

10 Ian Hodder, Reading the Past, Second Edition (Cambridge: CUP, 1991), 3; W. David Kingery, "Materials Science and Material Culture", Learning from Things, ed. by W. David Kingery (Washington: Smithsonian Institution Press, 1996), 181-203.

11 McGhee, "Ivory for the Sea Woman: The symbolic attributes of prehistoric technology", 59-66.

12 Colin Renfrew, "Varna and the Emergence of Wealth in Prehistoric Europe", The Social Life of Things, ed. by Arjun Appadurai (Cambridge: CUP, 1986), 141-168.

13 Stephen Mellor, "The exhibition and conservation of African objects: considering the nontangible", Journal of the American Institute for Conservation, 31, 1 (1992), 3-16.

14 William Morris, "Manifesto of the Society for the Protection of Ancient Buildings", Historical and Philosophical Issues in the Conservation of Cultural Heritage, 319-321. 
and the power of such artefacts is called emotive. ${ }^{15}$ Thus, the display of an executioner's sword in a museum may cause fear and horror in the viewer, while seeing the house where they were born will often create a feeling of nostalgia and joy in people.

\section{Contextual information}

The concept of "context" indicates the relationship between an object and its environment and forms a very important aspect of the informational structure of artefacts. Artefacts are created by the individuals or groups in a society where they form aggregates with other objects, which are used when performing specific tasks. Context can be described as a system that has two dimensions - material (physical) and conceptual. The physical context is comprised of the individuals and groups that have participated in the production and utilisation of the object, but also all the other objects related to object during the production process and the course of its future utilisation. The significant attributes of the material context also include the places and time related to the objects, as well as the activities and events for which the objects have been used or in which they have participated. For instance, these include the object's discovery site, which could be a geographical site or a room in a building, where the object was located. For instance, the physical context is formed of the parts of the environment that are closely related to the object, such as the soil of archaeological finds, or of other artefacts, such as the frame of a painting, the packaging or wrapper of an item, the burial places of other finds etc. In some cases, various objects comprise a set of objects, for instance, a set of china, suite of furniture, or furnishings of a specific room, etc. The physical context is very important in the case of different assemblages and collections. One and the same artefact can be the carrier of different meanings depending on the collection it is in. The value of the object may be restricted to the fact that it is part of a collection. Objects that are preserved in collections document not only themselves, but also the time and place where the collection was created. As a conceptual system, the context can be understood as a cultural and social environment that is related to the artefact. This can include the production system of the objects, the society's technological level,

15 Michael Brian Schiffer, Studying Technological Change: A behavioral approach (Salt Lake City: University of Utah Press, 2011), 23. 
division of labour, commerce, as well as beliefs, art, worldview, etc. In conclusion, it is difficult to say what the objects are not related to in the societies that have created them. A very important aspect of the conceptual context is the values related to the object. Objects are assigned definite values by society, which start to significantly affect their functions. It is possible to differentiate between the primary and secondary context. The primary context is the object's practical and utilitarian value. The primary context is determined by the following functions: preparation (furnishing, producing, preparing, creating); utilisation (consumption) and upkeep (maintenance, repair). In the secondary context, the artefact acquires documental value. An object that has previously had a primarily utilitarian value starts to be treated as a source of information. Above all, this means that a document becomes an archival document and an item becomes a museum object.

\section{Changing the informational content of artefacts}

The size of objects' informational content is not permanent, it changes constantly, since information is constantly lost and added. ${ }^{16}$ Any object that is treated as an artefact is the result of a historical process, and therefore, the "biography" of the objects to be preserved must be reconstructed in order to choose the best preservation strategy. Therefore, the diachronic states of the objects have to be added to the aforementioned synchronised list of data categories. The life story of an artefact always starts with the producer's idea, which is related to the producer's conceptual context, i.e. specific culture. There are no artefacts generally; there are only objects that originate from a specific culture and specific moment in time. The conceptual state of an artefact actually represents the potential object that exists at the idea level. The subsequent states of an artefact refer to a realised object immediately after the completion process has ended. In many cases, the factual state is only a hypothet-

16 Michael Ames, "Cannibal Tours, Glass Boxes and the Politics of Interpretation", Interpreting Objects and Collections, ed. by Susan Pearce (London: Routledge, 1994), 98-106; Arjun Appadurai, "Introduction: Commodities and the Politics of Value", The Social Life of Things, ed. by Arjun Appadurai (Cambridge: CUP, 1986), 3-63; W. David Kingery, "Technological Systems and Some Implications with Regard to Continuity and Change", History from Things, ed. by Steven Lubar, W. David Kingery (Washington: Smithsonian Institution Press, 1993), 215-230; Georg L. Miller, Olive R. Jones, Lester A. Ross, Teresita Majewski, "Approaches to Material Culture Research for Historical Archaeologists", Approaches to Material Culture Research for Historical Archaeologists, ed. by George L. Miller, Olive R. Jones, Lester A. Ross, Teresita Majewski (California, Pennsylvania: The Society for Historical Archaeology, 1991). 
ical construction since is it not possible to fix the exact moment when an object is completed - the producer may make changes; the object may remain unfinished; or it may be completed by someone else, etc. Throughout its "lifespan" the infrastructure of the object changes and the object reaches us in its actual state. The actual state includes both the initial information (primary data), as well as the information that has been lost and added during its utilisation, deterioration, conservation, etc. (secondary data). What is important is that the object in its factual state is not identical to the object at some later time. We may be dealing with the same painting, the same building, but this is no longer identical to the aggregate of physico-chemical attributes, functions, meaning as well as the context. Generally, ageing is understood to be a reduction of the informational level. Actually, in the course of aging, the informational value of an object may actually increase. Damage may add documentary value, by reflecting some important event and can also add aesthetic value, such as the patina, crackling or ageing of the varnish layer on paintings. The functional attributes of an object change exactly as the structural attributes do. Generally, the utilitarian value of an object decreases due to physical, technological and psychological obsolescence. If an object loses its practical value, it is often thrown away or reused as raw materials. Considerably more interesting is a situation where the decline in the utilitarian value of object increases it aesthetic and symbolic value. For example, a commodity that starts to be treated as a work of art whereby both its meaning and function changes. If an object ends up in a museum and is taken under governmental protection, its function and meaning change significantly. The object starts to be treated a document selected from the social and natural environment, i.e. from the primary context. Museum objects are objects that have been separated from the original (primary) context and been transferred to a museum reality in order to document the reality they have been removed from. By ending up in a museum all the information levels of an artefact end up losing information. The information at the structural level is affected by the physical preparation that the artefact undergoes when it is installed in a museum. Often the objects are cleaned and repaired with structural information being lost in the course of these activities. Part of this information loss is compensated by documentation. However, the previous functional and contextual information is almost totally lost (Fig. 1). The main challenge of preservation is preserving as much as 


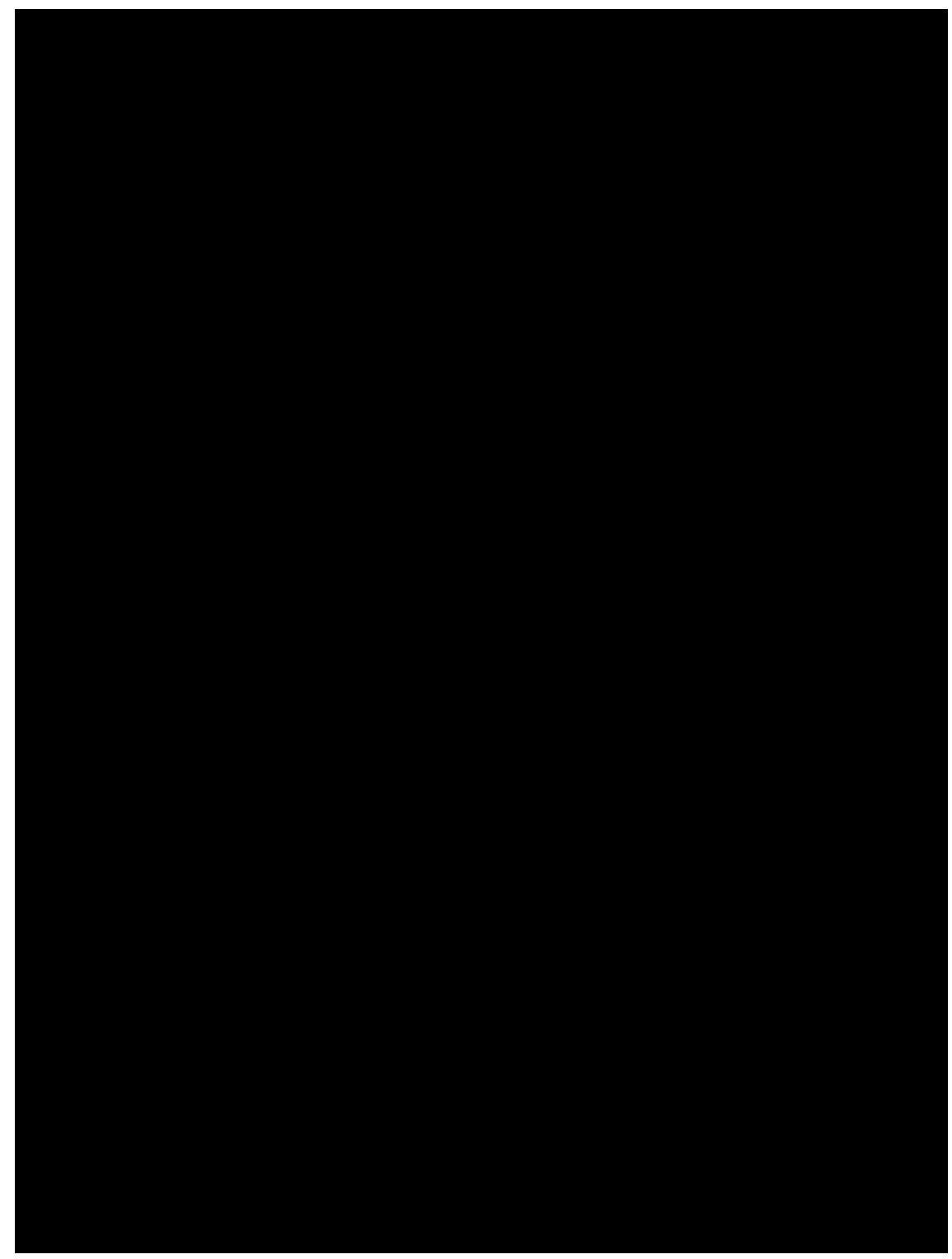

Fig. 1. When an object enters the museum all the information levels of an artifact will be changed dramatically. Plaster figures of Tartu University Art Museum. Photo: Kurmo Konsa.

possible of the artefact's informational structure. The item itself can tell us almost nothing about people and their culture. It is always necessary to try and preserve as much information as possible about the function 
and context of artefacts. A collection of objects is indivisibly related to its documentation. From the viewpoint of the artefact's informational structure, preservation is not a passive activity. The fact that an artefact ends up in a research institution be it a library, archive or museum changes its functional and contextual information. Not to mention the conservation or updating of information, which changes both the structural and function, as well as contextual information. When choosing a preservation strategy, considering the informational structure of the artefacts is fundamentally important and this applies especially to information updating technologies (creation of microforms, digitalisation, 3D modelling, etc.) in the course of which a new object is created with its own informational structure.

\section{VALUE-BASED CONSERVATION}

About 20 to 30 years ago, issues related to heritage values started rising to the forefront. Value-based conservation is based on approaches stressing values. The significance of objects is actually in the values and meanings that people attribute to them. An object or phenomenon is considered to be part of heritage only when certain values are attributed to them. These may be historical, scientific, aesthetic, artistic, social or some other values. It is values that make objects and phenomena into heritage. In this sense, values are conditional, because they are not objective like the weight, colour, chemical composition, etc. of an item. We cannot discover or define them and hope that they remain unchanged in the future. Values appear as the result of interaction between objects, contexts and people. John Ruskin and William Morris, the founders of modern conservation, also focused on the values of objects. They turned their attention to age value, artistic value, as well as educational and social value. ${ }^{17}$ Values as they related to heritage were dealt with scientifically for the first time by Alois Riegl in his Der moderne Denkmalskultus, sein Wesen und seine Entstehung, which was published in $1903 .{ }^{18}$ Values started to be examined more thoroughly again in the second half of the

17 William Morris, "Manifesto of the Society for the Protection of Ancient Buildings", Historical and Philosophical Issues in the Conservation of Cultural Heritage, 319-321; John Ruskin, The Seven Lamps of Architecture (New York, Dover Publications, 1989) 162-182.

18 See for example, Riegl, “The Modern Cult of Monuments: Its Essence and its Development”, 69-83. 
$20^{\text {th }}$ century and this primarily as they related to the economic significance of heritage.

\section{Heritage values}

The concept of values in connection with heritage is defined in two ways: as ethical principles or ideas, which provide rules of behaviour for both individuals and collectives; and as the principles used to compare the attributes that appear in objects, phenomena or people. Actually, both meanings of value are related to heritage. All the institutions or people dealing with heritage have their own values, which are also related to heritage. These values form the basis for people's everyday work and their impact on the management of the cultural heritage should never be underestimated. ${ }^{19}$ In the subsequent discussion, I will focus on values as they relate to the second field of meaning. Values are the properties attributed to objects, places and phenomena and the ideological and emotional connections related thereto, which make these objects, places and phenomena important and determine their significance for individuals, communities and societies. The objects and phenomena that are part of heritage have a large number of values based on which they are defined as being part of heritage. If these values do not exist, we are not dealing with heritage. Each object may have different values; different people and groups may identify with different values; and these values may change with time. Therefore, it can be said that no single universal immutable heritage value exists, but rather, heritage is comprised of a set of different values that are constantly changing. The aggregate of the heritage values typical of objects and phenomena is known as the heritage significance or cultural significance. The concept of values is used in the positive sense - if an object has value it is appreciated, has a purpose in society. Naturally these objectives may differ, as can the assessment of these objectives. Values that seem progressive and are appreciated by one group of people can seem negative and undesirable to another group. Several values can usually be highlighted for an object or phenomenon. Different people and interested parties can highlight different values and heritage can also be valued at different societal levels and from different viewpoints. Due to the large number of values relat-

19 Kriste Sibul, Kurmo Konsa, "Valuation of museum collections in Estonia: impact on conservation practice”, ICCOM-CC 16th Triennial Conference (Lisbon: Preprints CD, 2011), 1-8. 
ed to heritage, the process for defining values must also be pluralistic and eclectic. And it should involve various people and interest groups. The definition of the values related to heritage is not the prerogative of small groups of experts, since this approach contradicts the concept of a diversity of values. ${ }^{20}$ The value systems differ at the different levels of society. Yet, these value systems are all interconnected. Personal value systems develop based on what a particular person considers to be important. However these are not totally independent of the value systems at higher levels. People can adopt and accept some values, be opposed to others, or not pay them any attention.

Defining the value of objects is not a simple task. The values themselves are defined by different people differently and it is often quite complicated to draw a line between different values. Various value typologies must be used to define values. In order to create a typology, the values to be attributed to objects are divided into groups and each group is provided with a description. It is not possible to create a universal typology that would be suitable for all objects and phenomena in all situations. A typology is a necessary point of departure for defining values, but depending on the specific context, it must surely be adapted and, if necessary, augmented. Since values are dependent on their context, the values attributed to heritage by different people or groups of people can be contradictory or even conflicting. Heritage can also have different values depending on the social level. Values and value systems are not permanent, they are constantly changing and the values attributed to heritage objects and phenomena change along with them. New cultural activities and a changing context engender changes in heritage values. When defining heritage values, all the interest groups must be represented and the values important to them must be taken into consideration. One person cannot perceive all the values related to an object or locality. For instance, some objects of nature may have a spiritual or religious value for some people. The people who do not belong to this group may be ignorant of these values, and in some cases, these values are not revealed to those outside the group. Below, we provide a survey of the values that are most often associated with heritage.

20 Randall Mason, "Assessing Values in Conservation Planning: Methodological issues and choices", The Heritage Reader, ed. by Graham Fairclough, Rodney Harrison, John H. Jameson Jr., John Schofield (London, New York: Routledge, 2008), 99-124. 
Historical value is one of the most fundamental in case of heritage. More generally, this criterion reflects the historical development related to objects and phenomena. Historical value can be based on diverse aspects. The objects or phenomena may originate from the past, but this alone may be insufficient to give it historical value. Historical value reflects the relationship between heritage and people, event, places and themes. Objects and places may be related to famous people and significant events, but also to the everyday life of ordinary people. This criterion also definitely includes objects related to areas of activity, industry and lifestyles. These may be objects and localities that are typical of, as well as prominent or exceptional, unique or rare in, a particular time period.

Social value means that an object, locality or collection is considered important by a community in the present day. Social value is comprised primarily of the meaning that an object or locality possesses for people. There can be several reasons why the given objects, localities and phenomena are significant. For instance, these reasons can be spiritual, political, national or related to a sense of place. In any case, they are closely related to the community's identity and social cohesion. Sacred objects have special spiritual and religious meaning for certain groups of people. Often we are dealing with buildings and places that a particular social group considers as its own territory and which is related to the identity of that group. Such objects can be prominent sites, like the Tallinn Song Festival Grounds, but they can also be totally inconspicuous objects, such the bonfire grounds where a group of people regularly celebrates certain holidays. Such objects and localities characterise the longevity of traditions and permanence of culture. For example, ritual objects with social value, for instance flags with patriotic meaning, student fraternity caps and rapiers, etc., that are used when conducting certain ceremonies, and the fact they are used, gives them additional value. Social value is a current contemporary value. Very often the social value is combined with the historical and symbolic value. When the topical social value disappears, historical value may replace it.

Objects or phenomena acquire social value when a community feels that they are important. Social value is often actualised when some danger (rebuilding, demolition, etc.) threatens these objects or phenomena. Social value was identified for the first time in the Australian law of 
inheritance and the Burra Charter. ${ }^{21}$ When defining social values, the assessment must also reflect the views and attitude of the community for whom the objects or phenomena have this value. Museums, heritage boards or other institutions that organise the assessment of values cannot unilaterally define the value.

The spiritual or religious value expresses the sanctity of the objects and phenomena, their connection to the supernatural world. These can include grave goods, statues of gods, icons and sacrificial items, various ritual items and much more. These objects are located between the visible and invisible world. They have attributes that ordinary objects do not, but these attributes may not be reflected in their structural properties. These objects are often used in rituals and in places where rituals are conducted or are related to rituals. It should be taken into account that most religious works of art are not sacred objects, but rather record religious ideas. When dealing with spiritual values, we may come into conflict with other cultures. If we admit that values are social constructs then values are not intrinsic to objects. Yet, many other cultures do not share this position. For them, the sanctity of the objects and phenomena are intrinsic. In Europe, the idea of the actual existence of sanctity (God, saints) in objects and places lost its validity after the Protestant Reformation, but, for example, in Catholicism and very many native religions, some objects are holy regardless of whether anyone believes it or not. The spiritual value does not need to be linked to any specific religion or its acknowledgement by people. Certain objects, localities and phenomena also provoke respect, wonder and a sense of solemnity in secular people. Such feelings can be inspired by things created by humans, as well as natural objects and phenomena of nature. Some examples are churches, cemeteries, prominent natural objects, etc. Both things created by humans and natural objects and landscapes can be sources of inspiration for people. This can be expressed directly in works of art, for example, Montagne Sainte-Victoire inspired Paul Cézanne to create several paintings.

Aesthetic value is a very significant heritage value, which can be comprised of several parts and interpreted very differently. One of

21 Australian Heritage Commission Act (1975), http://www.unesco.org/culture/natlaws/media/pdf/ australia/australia_act_n2_1975_eng_orof.pdf (retrieved on 14.11.2015); The Burra Charter (Burwood: Australia ICOMOS Incorporated, 2000), http://australia.icomos.org/wp-content/uploads/BURRA_ CHARTER.pdf (retrieved on 14.11.2015). 
the most obvious uses is related to the visual attributes of objects. The beauty category has often been one of the very important reasons for including an object to be part of heritage. More broadly, aesthetic value is related to all kinds of sensory experiences that heritage provides. An object can be aesthetically valuable due to its artistic processing, style, technical mastery or beauty. The object can originate from folk art or fine art, be unique or mass-produced. The aesthetic value is most obvious in art works, handicraft items and decorative works. However, in order for an object to have aesthetic value, it does not need to be a work of art. Minerals, fossils and other natural objects can also have aesthetic value. And, buildings, landscapes and parts thereof, can also have aesthetic value. And one also speaks of landscape panoramas or scenes that should be preserved.

Quite a large number of the things that are preserved as cultural heritage have artistic value. Objects and phenomena that are considered to be art have artistic value. However, what is considered to be art depends on the context. Much of what is currently considered art was not art when it was created. Artistic value is closely related to aesthetic value, but they are not inseparable. All beautiful objects are not necessarily art and many art objects have no aesthetic value, at least for many people. Artistic value is closely related to historical value. The works of famous artists or architects often have artistic value and as do the works that represent some important style of art.

Objects and phenomena with symbolic value symbolise something; have significant meaning for the society and individuals; and function as certain symbols and/or texts. Very often, we are dealing with national, governmental or religious symbols that allude to a glorious past. For different people, a specific heritage item can symbolise different things. In some cases, the different interpretations can co-exist without any problems; in other cases, they are antagonistic and can cause or aggravate tensions between different groups of people. Intrinsically, the connection between a person and a place gives it symbolic value. A homeland, home village, place of birth - these allude to the symbolic value of certain places. Similarly, a place that is related to a community has symbolic meaning for that community. Natural landscapes comprise one part of a nation's idea of its homeland, and as such, they have significant symbolic value. Symbolic value provides context and mean- 
ing to everyday life, characterises the given region, and the distinctive traits of the people.

Actually, all the values related to heritage are political, since the heritage process itself is political by nature. ${ }^{22}$ More narrowly, political values express and represent a certain type of social system and the political views related thereto. Often they are considered to be part of the symbolic value, since political value can be attributed to the most diverse objects, localities and phenomena. Political value, like the other values, is temporally variable.

Scientific or informational value is related to the information found in objects, localities or phenomena and its use as a research resource. An object or collection has scientific value when it has great potential for future research work. For instance, archaeological objects and collections, biological and geological collection, as well and documentary archives are scientifically significant. In the case of scientific significance, it is extremely important to know the exact context and to have proper documentation. At least potentially this value exists in all objects per se, because they can be used in the research of the history of materials and technology, etc. The historical environment as a whole is a very important source of knowledge about our ancestors and their societies.

Use value is characterised by the fact that objects and phenomena can help in the interpretation of events, experiences, historical topics, people, structures, regions and highlight their various aspects. This value reflects the value of objects and collections from the standpoint of exhibitions, educational programmes, etc. at informational institutions; and also due to its connection with collectible topics, the history of the collections and the way they are interpreted. Objects and collections in situ are important for demonstrating the importance of places and because of their connection to people. Some inherently an ordinary and unimportant item can be significant if it alludes to some historical event or topic. Usability is important in the case of work and measurement tools, which can be used to demonstrate certain methods. Landscapes, urban environments and parks where people go to rest and relax can have use value. These pleasant and comfortable places are very often environments with historical value. In this case, we can also speak about recreational value. Often, use value is based on something else, for in- 
stance, historical value. The skilful interpretation and presentation of the heritage is necessary for the realisation of the use value.

The sentimental value originates from a person's direct personal experience with the objects, phenomena and places. Objects usually have this value as long as the person that values them is alive; but some objects are handed down from generation to generation. This is a value that is characteristic of the personal and familial level.

Commemorative value is important in the case of memorials and is based primarily on the objectives of those erecting the object at that time the monument is created. According to Riegl, these objects aspire to immortality and eternity. The objective is to keep past events alive and preserved in our memory. ${ }^{23}$ From the standpoint of commemorative value, the objects may, and even should, look new, since historical layers are not significant in the case of this value. Objects with this value do not need to be located in the places where the events to which they are dedicated took place. Examples are various war memorials.

Objects with associational value are related to prominent people, places and events or groups. These objects reflect their contemporary situation. However, they may not acquire historical value.

An object has newness value when it looks new and when looking new is viewed as an attribute. For instance, collectible coins, books and other objects have newness value. In the case of old objects, the opposite may be true and looking new after cleaning or conservation may be a negative attribute.

An object has age value when it looks old, is old and also when looking old is viewed as an attribute. Such objects should not be processed in order to erase the external manifestations (e.g. crackling on paintings, patina on objects) of aging.

Rarity expresses the value of an object that is rare, unusual or a splendid example of a specific type of item. It could be a unique, handmade object, or one with special decorations. Or it could have other attributes that distinguishes it from other similar objects. Other values should accompany rarity. After all, there are objects that are rare but no one considers them valuable. Rarity strengthens other values and increases their importance. When we have an item with historical value that is also rare, the value of the object is further increased.

23 Riegl, “The Modern Cult of Monuments: Its Essence and its Development”, 69-83. 
Integrity or authenticity value is reflected in the object is whole, i.e. has not suffered great damage, is complete and in its initial, original or very close to its original state. And the object should have no intentional or unintentional changes that prevent it be perceived as original. This does not mean that, in order for this criterion to apply, the object must be in its initial or original state. Changes, reconstructions, traces of wear, etc. may actually increase the importance of the object, as they are part of its history. However, it is clear that a piece of furniture that has preserved its original finish is considerably more valuable based on this criterion than the same object that has been restored. If an object is comprised of various parts or a set of objects is involved, all the parts and items should exist. This criterion also includes collections that have survived as a whole and buildings that are an important part of a building ensemble.

Provenance reflects the existence of information regarding the context related to the object or collection's owners and use. This is a very important additional criterion for determining historical or scientific value. The documented history of the object's existence, utilisation and owners provides the object with a context for the society or individual. For example, the provenance of works of art and archaeological finds as well as archival documents is extremely important.

The economic value of cultural heritage is divided into use value and non-use value. The latter is in turn divided in three: existence value, i.e. people value the existence of the heritage, but they cannot actually use it; option value, i.e. people want to preserve the option that they or others may be able to use the cultural heritage in the future; and bequest value, i.e. people want to bequeath it to future generations. Use value is related to the financial benefit to be gained from the heritage at the present time.

The monetary or market value is related to the current market value of the object and this may change very rapidly. The monetary value is not directly related to the other values. Monetary value cannot be used to characterise all the other values (if something is expensive, ergo it is very valuable in every sense).

The evidential value or legal value refers to the value of objects, places and phenomena as proof of the provenance of their creators, their functions and activity. This allows the facts and occurrence of events 
to be proven. Although usually associated with archival documents, all kinds of heritage can have evidential value.

Administrative value refers to an object's significance from the viewpoint of ensuring the activities or functions of an organisation. For example, architectural drawings and plans for the repair of buildings, maps to confirm changes in the landscape, ecosystems and heritage objects, etc.

\section{Associating values with the conservation process}

According to value-based approaches, conservation is treated as a social process, the objective of which is to ensure the preservation of values with the help of direct processing. The goal of conservation is to stabilise the state of the elements that bears the object's values. Depending on the objects and the goals of the processing, very different physical and chemical methods can be used to achieve this. An attempt is made to slow down the decrease in the values as much as possible. The term "restoration" indicates an activity with the goal of restoring the object to its presumable state at some earlier time period. The values characteristic of the object at that period in time must be so important that they outweigh the reduction of other values. During restoration, changed or damaged elements may be removed and new ones added. Restoration is based on comprehensive research about the object and its history (Fig. 2). Restoration increases the aesthetic and use value, but the scientific value may be reduced. Therefore, objects in which the scientific value is primary, such as archaeological finds, are generally not restored.

In the case of a value-based approach, the views and values of the groups of people related to the heritage is taken into account, but the material aspects are still the focus. The decision-making processes related to conservation are managed by experts, but other interest groups are also involved, for example, local people, artists, creators of heritage objects, owners, users, museum employees, other conservators, etc. This type of value-based approach has clearly gained a firm footing in conservation today. The physical integrity of objects is still central, but the cultural significance of the objects is also considered and included as a significant component in the decision-making process. Since the condition of the object, as well as the cultural values, must be taken into account, the decision-making process often involves various specialists and shared decision-making processes are also spoken about. 


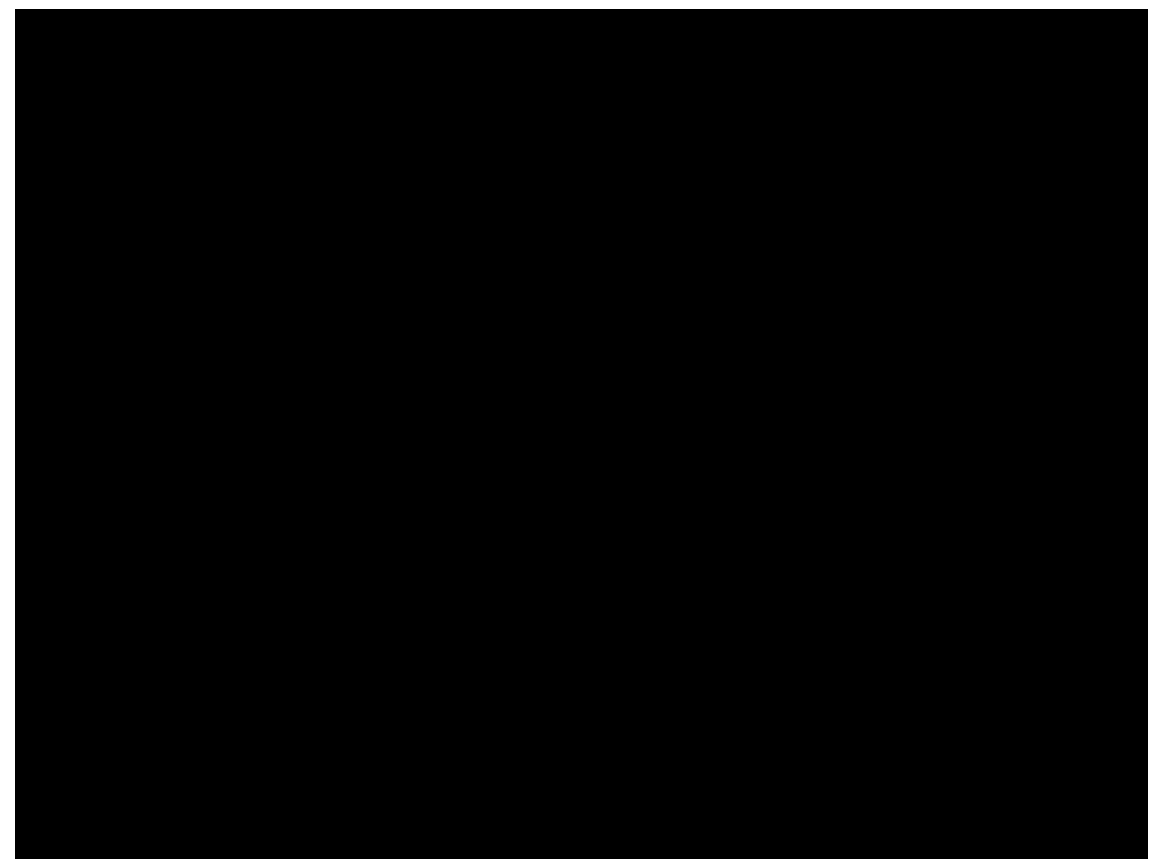

Fig. 2. The important part of conservation process is the study of objects. Ülle Vahar, the chief conservator at Estonian National Museum studies the chest. Photo: Kurmo Konsa.

However, one problem arises when dealing with values. Values are not inherent to objects; they develop during the heritage process and depend on the context. When we are assessing the values of an object, it is not possible to assess all the values that a given object may possess for the people that are related to it. Thus, the determination of various values is always incomplete and partial, being limited by time, resources and the skills and knowledge of the assessor. However, what is the objective of conserving and preserving heritage more generally? In brief, conservation must be related more to the present day and to the people that give objects their meaning. And these are contemporary people. The people from the past are dead and we can research their values and meanings. And it is fundamentally impossible to know what the values of people in the future will be. Thus, all that's left is contemporary people. The new approach to conservation that put people at the centre of the entire process is known as people-based conservation. 


\section{PEOPLE-BASED CONSERVATION}

Heritage is linked to people's lives in many ways and the name of this approach alludes to this fact. And conservation must strengthen and promote this connection in every possible way. Attention is shifted to how the conservation process and its results affect people. The focus is no longer on the material object as the carrier of values, but on the community that attributes these values to the object. Maintaining and forming the wellbeing and values of contemporary communities manages the entire conservation process. Before starting to conserve a building or other heritage object, one must understand the conception of the object and the values related to the object held by interest groups and communities. This approach recognises the locality, subjectivity and political nature of conservation. The values of objects are created by groups of people. Values are dependent on context and are shaped by contemporary communities. Conservation depends on the personality of the conservator, his or her training, beliefs and sensitivity. We always choose certain values and ideas that are embodied by the given object and which we want to preserve. Other values and ideas are discarded. In other words, we choose what we want to present to people and to preserve.

During conservation, the social networks that unite the objects with meaning and value to people and societies are processed rather than material objects or non-material objects that have values. How is this achieved? How can the conservation process be linked more strongly to society? This is a complicated question. And a suitable answer is still being searched for. What is clear initially is that in order to find the answer, we need to turn our gaze away from conservation and try to find approaches that can link people, values and activities. One such approach is information ecology. Information ecology is an information science that uses the concepts of ecology to analyse complication information systems by viewing them as ecological systems. ${ }^{24}$ An information ecology system is a complex of the people, activities, values and technologies in a specific local environment. An information system is an integrated network of a group of people and their tools and activities. An approach

24 Bonnie Nardi, Vicki O’Day, Information Ecologies: Using Technology with Heart (Cambridge: The MIT Press, 1999); Thomas H. Davenport, Information Ecology: Mastering the Information and Knowledge Environment (Oxford, New York: Oxford University Press, 1997). 
based on information ecology is characterised by systematisation, diversity, co-development (co-evolution) and locality.

The word "ecology" is familiar to people today but it is associated mostly with environmental problems, nature preservation, natural resources, back-to-nature lifestyles and the impact of humans on nature more generally. Less familiar is the use of the word ecology in connection with other fields of activity, for example, behaviour (behavioural ecology), ethics (ecological ethics), the economy (economic ecology), culture (cultural ecology), psychology (ecological psychology) or settlement geography (settlement ecology), to name just a few. It seems that ecological approaches have something to offer in other fields of activity as well. So, what makes ecological thinking so attractive? And is it truly a meaningful approach or just a fashionable term that is applied to a variety of things in order to attract attention and advertise them. The explicit definition of ecology is a science that deals with the interactions among organisms and their environment. However more generally, in the case of an ecological approach, a living or lifeless system is dealt with as a whole, by examining the interactions between the system's elements as well as the relationships of the system as a whole with the external environment. In the case of lifeless systems, the concept of ecology is metaphorical, creating an image of a biological environment, along with the complicated dynamics, diversity of species and ecological niches characteristic therein.

Information ecology is a system of people, activities, values and technologies in a definite local environment. However, the attention is not focused on technology, but on the human activities supported by technology. The idea of an information ecology approach is to focus on the interactions between technology, people and their activities. Such an approach is based very clearly on the principle that any research must be directly related to practices. The ecological metaphor should promote thinking and discussions and ultimately result in more successful actions. Significant keywords, which characterise the information ecology approach, are value-based, systematisation, co-development and locality. Below, we will take a closer look at this approach and show how the principles are implemented in heritage practices. 


\section{Value-based}

The implementation of information ecology principles is based on the system's fundamental values and meanings, which the user attributes to the activities and techniques. An integrated information ecology system is characterised by the use of technology in a social network comprised of values, standards and customs. Social practices are the essential element in an information ecology system and do not provide technologies, but ways to use them. The development of an integrated information ecology system must be based on values that help to prevent internal contradiction that may cause the collapse or non-implementation of the entire system. The design of a technological system is usually based on efficiency and performance, but if the created system contradicts people's ideas and values, considerable stress can develop in the system.

It is most important to consider the values related to heritage whenever making any decisions impacting heritage. Heritage is always the carrier of values and meanings. Working out these values and taking them into account forms the basis for the management of the entire heritage process. One proceeds from what the society values and why. According to the contemporary approach to heritage, the physical and spiritual aspects of heritage are inseparable. However, in practical heritage management, the main focus continues to be on the material side of heritage. And there is clear reason for this, since the material side of heritage is easier to perceive and manage. The basis for management is the clear definition of heritage values. All objects and phenomena are not equally valuable and it is not possible to manage everything. Heritage management always means dealing with problems and objectives. These are often contradictory and they need to be ranked by priority, balanced and compromises found. Objectives that are related to the definition of values manage the entire heritage management process.

\section{Systematisation}

The systems involved in information ecology are complicated, but do not have the complexity of large systems to which system theory can be applied. In the case of an ecological approach, the view of the system is based on people. We all belong to and participate in many different systems. The ecological dimension enables the contact points between the individual and the system to be identified, along with ways to penetrate the system and possibilities for influencing the system. Similarly to bio- 
logical ecology, strong connections and interactions between the various parts of the system also characterise information ecology. A change in the ecological system usually involves the entire system - when one element of the systems changes, repercussions felt throughout the system. It is important to make sure that the approach to heritage involves all the dimensions of heritage - the various objects and phenomena, various social levels and various values.

\section{Co-development (co-evolution)}

An integrated ecosystem is always changing; it is never static, even in a stable state great changes are often taking place. The development of technology means that new, different, more effective tools and services are constantly being offered. People must be prepared to participate in the development of the information ecology systems that surround them. Since technological changes are constant, information ecology systems must also change and adapt constantly. People, who learn, adapt and create information, are part of the information ecology system. Even if technology does not change, people's ability to deal with and use it improves. The social and technical aspects of the environment develop coherently - co-evolutionarily. Comparably to natural ecosystems, "key species" are also important to informational ecosystems. In ecology, species are defined as key species if their existence and activity is necessary for the preservation of the entire community. If key species are destroyed or lacking, the entire community perishes. In information ecology, these "key species" are the respective skills and experiences of people, which are necessary for the effective use of technology. These are the mediators, people who are able to interpret information, turn data into knowledge and make it comprehensible to others. People are inevitably the most important parts of every information ecology system. Unfortunately, very often the activity of these mediators is informal and insufficient attention is paid to this; and it occurs on the periphery of the system. Yet it is absolutely clear that the success of implementing new technologies depends on these mediators who are able to adapt the technologies to the existing local conditions. The central idea in the preservation of heritage is the management of change, not only the preservation of physical material that originates from the past. The management of change means that it is not possible for us to preserve objects and phenomena if it is separated from their 
physical and social environment. Since this environment is constantly changing, heritage management must adapt to these changes.

\section{Locality}

In the case of information ecology approach, special attention is placed to the issue of locality. People always function in a definite place; all contacts with technology also take place in a definite place and these places are certainly not unimportant. As unbelievable as this may seem to us, technology is always different under different local conditions, since the presumable roles, usability and other attributes of specific technical solutions always differ. Namely, in most cases, the success of technology also depends on being familiar with and perceiving a specific place. Heritage is society-specific and inherently political. We cannot remove heritage management from the social and political environment. It is important to consider the views, goals and expectations of the community and society where the given heritage item is located. It is definitely necessary to involve as many interest groups as possible in the management process. Connectivity with the public and the support of the society are essential for the successful long-term preservation of heritage.

In heritage management, after the emergence of the value-based approach in the second half of the 1990s, a participatory process with the involvement of various views and interest groups has started to be preferred over a top-down approach. In this way, it is possible to increase the number of people interested in heritage and ensure more sustainable heritage management. ${ }^{25}$ Traditionally, experts are the ones making heritage-related decisions. We cannot involve other interest groups if they lack the actual right to make decisions in the heritage management process. This means that the interest groups related to specific heritage must have the same right to make decisions as the experts. In heritage management, it is not possible, or necessary, to aspire to perfect results, it is only important to achieve the best possible result, based on the local conditions.

25 Kate Clark, Conservation Plans in Action: Proceedings of the Oxford Conference (London: English Heritage, 1999). 


\section{Information ecology and the conservation process}

So, how can we use information ecology to more successfully link the conservation process to society? If we summarise the concepts related to information ecology from the viewpoint of the conservation, we need to emphasise the importance of a systematic approach. According to the traditional conservation model, the real world (object) is interpreted by the conservator using scientific methods. Thereafter, the object is changed and then it is reinterpreted by the user. This is an objective model that is untouched by any subjective, personal approach. In the case of a more complicated and personified model, all the participants in the process have their own worldviews, which comprise the intellectual, philosophical, experiential and social aspects related to their knowledge, everyday life and experiences. The worldviews related to individuals, professions and institutions differ from each other. Based on the differences in worldview, the perception and interpretation of objects also differs. Objects, in turn, are impacted by people. By conserving different objects we are continually learning and developing our knowledge and skills. Therefore, people's worldviews are also constantly changing and developing. And therefore it is not possible to expect conservation rules or situations to be objective. The opinions, beliefs, experiences, etc. of the participants in the process influence the decisions made about conservation (Fig. 3). This model reflects a subjective interpretation rather than an objective explanation. Thus, conservators, the owners of objects, as well as the broader public, to mention a few of the parties, comprise an integrated system. ${ }^{26}$ Inevitably, the various parties tend to view this system differently. On the one hand, this is unavoidable, but on the other, it is an obstacle that needs to be surmounted. When dealing with the importance of conservation, besides the often prevalent economic importance, we need to consider its role in shaping identity and creating social cohesion and the part it plays in education, and more generally, in the social communication system. ${ }^{27}$ By conserving objects, meanings are created and values presented to the community and society more generally. An important factor that needs to be stressed is the locality and uniqueness of heritage. Every heritage object and phenomenon is located in a definite environment and is inseparable from it.

26 Dean Sully, Isabel Pombo Cardoso, "Painting Hinemihi by Numbers: Peoples-based conservation and the paint analysis of Hinemihi's carvings", Studies in Conservation, 59, 3 (2014), 180-193.

27 Kurmo Konsa, "Milleks meile pärand?", Akadeemia, 12 (2013), 2171-2189. 


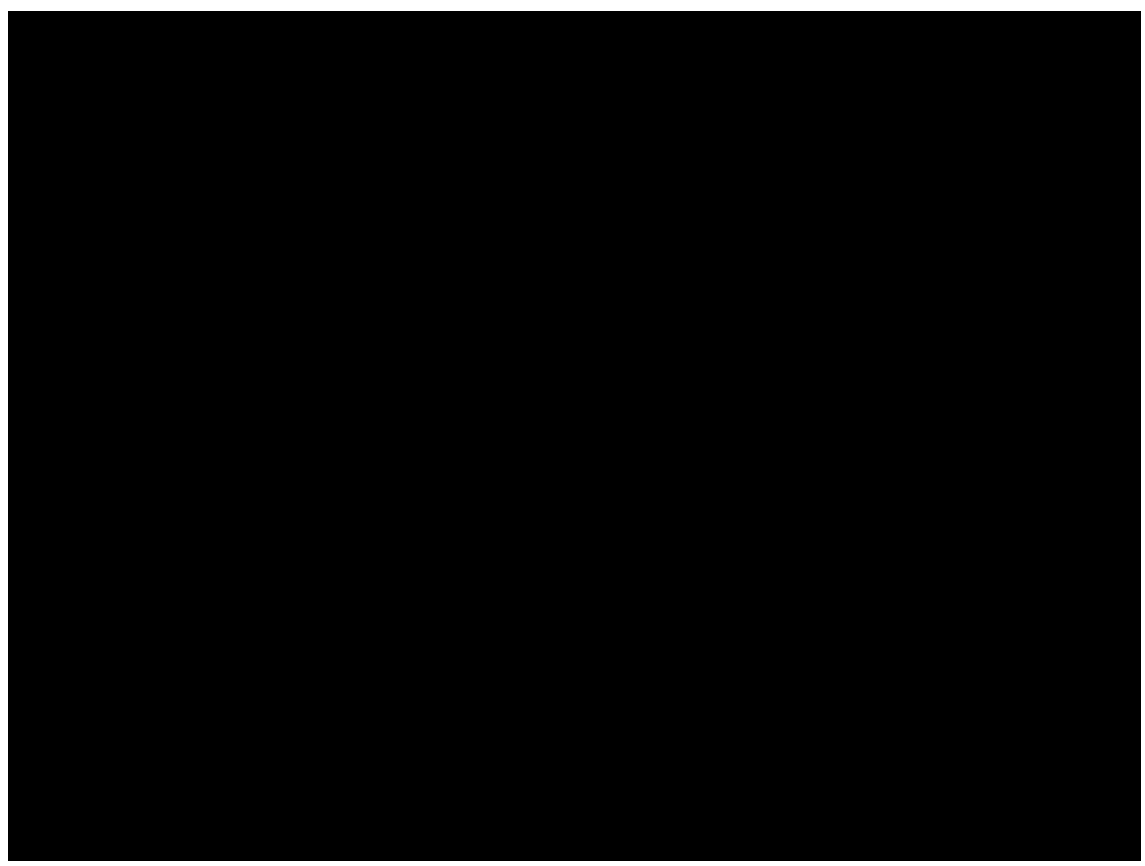

Fig. 3. Conservation combines both manual operation as well as collective decision-making. Conservators cleaning the old forge bellows at Mõniste Museum. Photo: Kurmo Konsa.

\section{SUMMARY}

Heritage is both a part of physical reality and a spiritual phenomenon. Heritage links people to each other and to the environment, with both its material and wildlife aspects, and therefore, is a part of our world. Sustained by heritage, by recreating it again and charging it with significant meanings, people shape the functioning of societies. Meanings are created through heritage and values are presented to communities and the society in general. I believe that this participation in the creation of value environments ensures a place for heritage in today's information culture world and the future world of artificial culture. The management of heritage also includes the conservation of objects. Conservation changes the technical processing of objects into a way for creating and recreating culture. What can be achieved with conservation is important, but so it how it is done, and how it influences people. Conservators choose objects and conserve one possible future. They should choose one that people will enjoy living in. 
Kurmo Konsa: Modern Conservation: Connecting Objects, Values and People

KEYWORDS: HERITAGE; CONSERVATION; OBJECT-BASED CONSERVATION; VALUE-BASED CONSERVATION; PEOPLE-BASED CONSERVATION; INFORMATION ECOLOGY

\section{SUMMARY:}

Heritage is a technique that we use today to create the present and the past and it depends on our current choices rather than on the past. Heritage is only a tool, but we should not forget that it is a very powerful tool for making these choices and implementing the decisions based thereon. The goal of this article is to connect the main approaches to conservation (object-, value- and people-based methods) with the corresponding information models. I propose that information content models are central to object-based conservation and naturally value-based conservation relies on typologies. And I associate people-based conservation with ideas related to information ecology. One way to preserve the objects that are part of heritage is to conserve them. Conservation as a profession as we know it today developed during the $19^{\text {th }}$ century. Along with the processing methods, various theoretical approaches, which provide the reasons for and explain the circulation of the heritage objects, are very important in conservation. The conservation approaches can be defined as being object-, value- or people-based, according to their focus. These approaches are not used in a definite temporal sequence, whereby they would preclude each other. Depending on the context, a specific temporal sequence does exist, but they are all in use today. The choice of the preferred approach depends on the goals of the method. These approaches express an increasingly inclusive and complex approach to conservation. Today, the treatment of objects as phenomena related to information is at the centre of the conservation theories for object-based conservation. Value-based conservation relies on various value typologies. And it is these values that change objects or phenomena into heritage. The new approach to conservation that put people at the centre of the entire process is known as people-based conservation. How is this achieved? How can the conservation process be linked more strongly to society? This is a complicated question. And a suitable answer is still being searched for. What is clear initially is that in order to find the answer, we need to turn our gaze away from conservation and 
try to find approaches that can link people, values and activities. One such approach is information ecology. Information ecology is an information science that uses the concepts of ecology to analyse complicated information systems by viewing them as ecological systems.

CV:

Kurmo Konsa is an Associated Professor at the Department of Archival Studies at the University of Tartu, and Professor of Conservation at Tartu Art College. He owns an MSc in microbiology from the University of Tartu, and an MA in Book Science from Tallinn University. Kurmo Konsa holds a doctoral degree in Informational Science from Tallinn University. His doctoral work focuses on preservation of written heritage and preservation surveys issues. In the past he has worked at Tartu University Library as paper conservator and at Estonian Postal Museum as conservator and curator of collections. 